\title{
ELECTROMECHANICAL MODELING OF THE HUMAN ATRIA
}

\author{
Fritz $\mathrm{T}^{1}$, Dössel $\mathrm{O}^{1}$ and Krueger $\mathrm{M}^{1}$ \\ ${ }^{1}$ Institute of Biomechanical Engineering, Karlsruhe Institute of Technology, Germany \\ Thomas.Fritz@kit.edu
}

\begin{abstract}
Cardiac computer modeling can help to gain a deeper insight into the physiological processes of the heart. In this work we present a new electromechanical modeling framework which allows to simulate the contraction of the atria in a model of the whole heart with realistic boundary conditions. For the active tension development (TD) we used a model, which was originally developed to describe the TD of the ventricles. However, TD in the atria differs significantly from that of the ventricles. On that account, we adapted the TD model to the measurement data of the atria. The modeling framework allows to obtain a realistic motion of the atria during the contraction cycle.
\end{abstract}

Keywords: Electromechanical Modeling, Atria, Active Tension Development

\section{Introduction}

In this work we present a new electromechanical modeling environment which allows to simulate the contraction of the atria within a whole heart model. It consists of four components: a geometrical model of the heart, an electrophysiological simulation environment, an TD model and a biomechanical model. The TD model, which was initially adapted to the ventricles was adjusted to measurement data of the atria.

\section{Methods}

Geometrical model: The geometrical model of the heart included a high resolution hexahedral mesh of the atria and a coarser tetrahedral mesh of the whole heart including the ventricles, the atria and a thin layer surrounding the heart representing the tissue in which the heart is embedded. The hexahedral mesh was used for the electrophysiological simulation while the tetrahedral mesh was used for the biomechanical simulation. For both meshes, fiber orientation was defined. For the ventricles, the rules of Streeter et al. [1] were used, while for the atria, the method presented by [2] was used.

Electrophysiological modeling of the atria: The electrophysiological environment employed the Courtemanche et al. (CRN) model [3] to describe the electrophysiological processes on cellular level. Moreover it provided the intracelluar calcium concentration, which was the input for the TD model.

Adaption of the TD model to measurement data of the atria: The Hybrid Tension Development model (HTD) presented by Sachse et. al [4] was used to to calculate the contractile forces. It is based on the sliding filament theory which is modeled using three Markov chains. The first one describes the binding of calcium to troponin $\mathrm{C}$ depending on the calcium concentration provided by the CRN model. The second Markov chain describes the configuration change of tropomyosin due to bond calcium, while the third Markov chain describes the cross-bridge cycle including the interaction of actin, myosin and ATP. The parameters of the HTD model were initially adapted to the TD of ventricular myocytes. However, the TD in the atria is faster than in the ventricles as shown by $[5,6]$. For that reason, we used an optimization algorithm (Powell's method) to adapt the HTD model to the measurements of Schotten et al.. In order to reduce the parameter space, prior to this, we conducted a sensitivity analysis of the HTD model to determine those parameters which have the most significant influence on the morphology of the TD curve.

Biomechanical model: The biomechanical model was based on a continuum mechanical approach. The passive mechanical properties of the heart were described by the model of Guccione et al. [7], while the contraction was driven by the TD which is provided by the adapted HTD model. Although the ventricles did not contract actively during the atrial activation, they limited the motion of the atria and thus allowed to obtain a more realistic description of the deformation of the atria. As boundary conditions, the atria were defined to be fixated at the openings of the venae cavae and the pulmonary veins and the ventricles at the apex. Additionally, a contact handling algorithm was used to enforce permanent and frictionless contact of the heart with the surrounding tissue.

\section{Results}

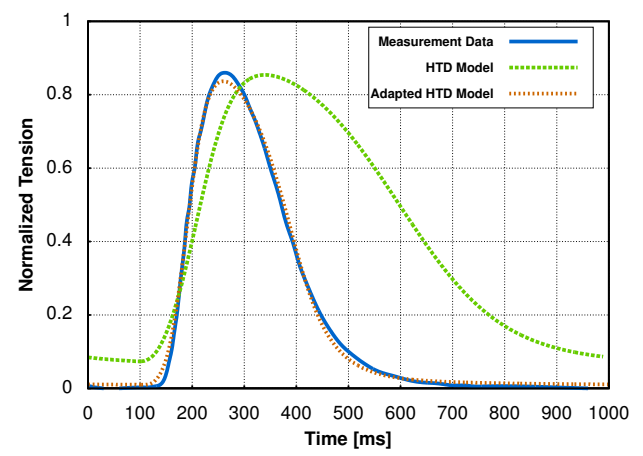

Figure 1: Measured TD (blue), TD obtained from the original HTD model (green) and from the adapted HTD model (orange)

Fig. 1 shows the measurement data of Schotten et. al and the TD curves obtained from the original HTD model for the ventricles and from the adapted HTD for the atria. 


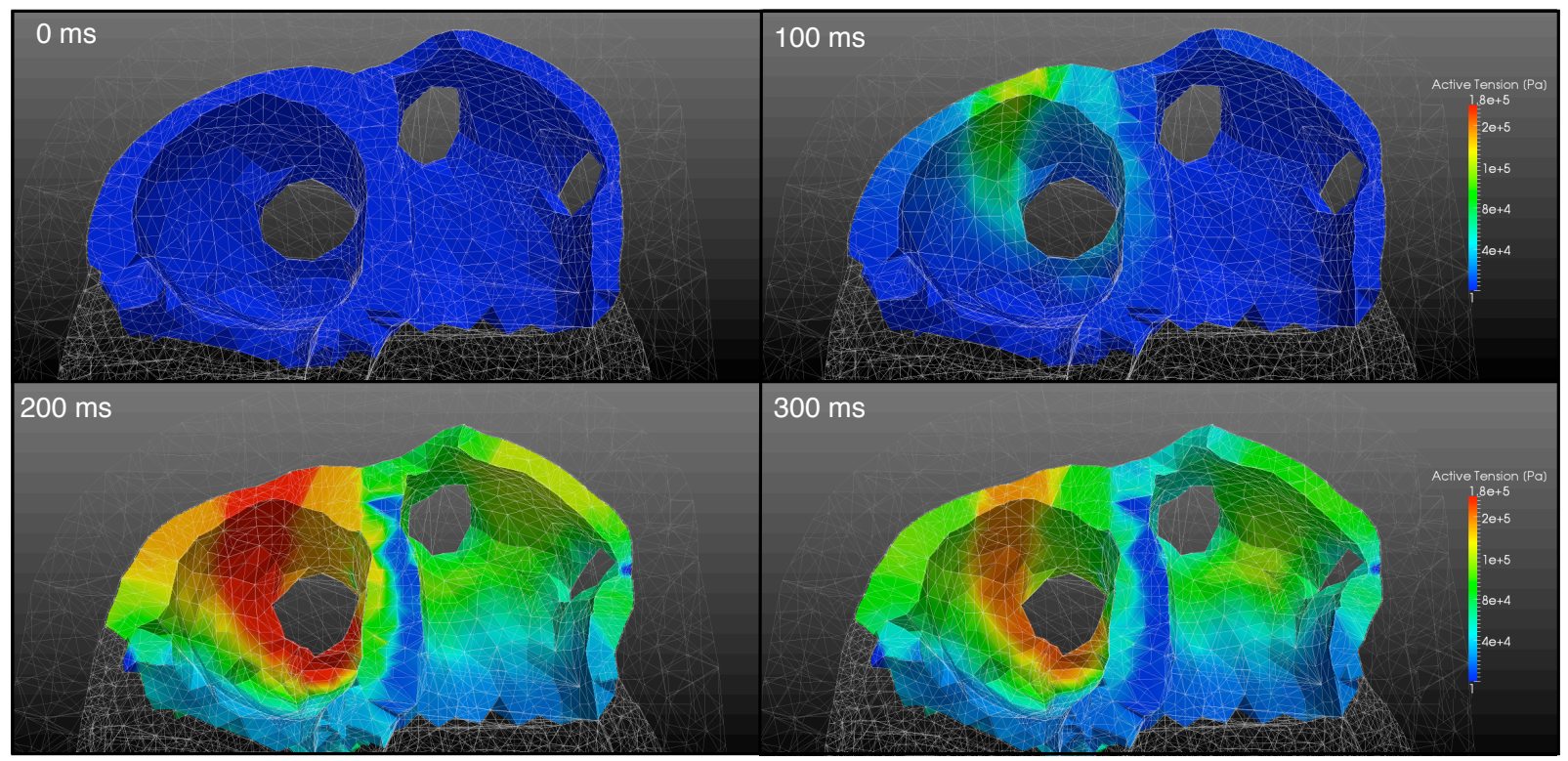

Figure 2: Sequence of four simulation time steps of the atrial contraction with color-coded TD. The wireframe represents the ventricles and the surrounding tissue.

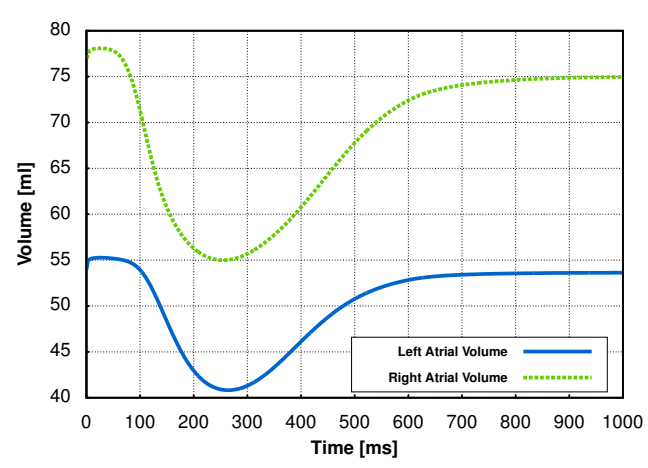

Figure 3: Volume curve over time of the left (blue) and right (green) atrium

Hereby, the measured data curve and the curve of the adapted model were normalized to the maximum of the original HTD model. The final root mean squared error between the measured atrial TD and the adapted HTD model after the parameter optimization was $1.68 \cdot 10^{-2}$. Fig. 2 shows a sequence of four time steps of the simulation of the atrial contraction. The TD is initiated at the sinus node and propagates over the whole atria. Figure 3 shows the volume curve of the left and right atrium during the atrial contraction. At maximum systole the left atrial volume is decreased by $26.1 \%$ and the right atrial volume is decreased by $29.6 \%$.

\section{Discussion}

The adapted HTD model, which was obtain from the parameter optimization showed a good match with the measurement data. In combination with the biomechanical model, it enabled the simulation of the contraction cycle of the atria and to predict the atrial volume curve. However, in the current simulation framework, no blood flow in the atria is taken into account, yet. The atrial contraction supports the filling of the ventricles and increases the ventricular efficiency (atrial kick). One application of the computer model could be the optimization of ablation patterns for the treatment of atrial fibrillation regarding their impact on the contraction of the atria.

\section{Bibliography}

[1] D. Streeter, "Gross morphology and fiber geometry of the heart," Handbook of physiology, pp. 61-112, 1979.

[2] M. Krueger, V. Schmidt, C. Tobón, F. Weber, C. Lorenz, D. Keller, H. Barschdorf, M. Burdumy, P. Neher, G. Plank, et al., "Modeling atrial fiber orientation in patient-specific geometries: a semi-automatic rule-based approach," Functional Imaging and Modeling of the Heart, vol. 6666, pp. 223-232, 2011.

[3] M. Courtemanche, R. J. Ramirez, and S. Nattel, "Ionic mechanisms underlying human atrial action potential properties: Insights from a mathematical model," Am. J. Physiol., vol. 275, pp. H301-H321, 1998.

[4] F. Sachse, K. Glänzel, and G. Seemann, "Modeling of protein interactions involved in cardiac tension development," $I J B C$, vol. 13, no. 12, pp. 3561-3578, 2003.

[5] U. Schotten, S. Verheule, P. Kirchhof, and A. Goette, "Pathophysiological mechanisms of atrial fibrillation: a translational appraisal," Physiol Review, vol. 91, pp. 265-325, 2011.

[6] U. Schotten, M. Greiser, D. Benke, K. Buerkel, B. Ehrenteidt, C. Stellbrink, J. F. Vazquez-Jimenez, F. Schoendube, P. Hanrath, and M. Allessie, "Atrial fibrillation-induced atrial contractile dysfunction: a tachycardiomyopathy of a different sort," Cardiovasc Res, vol. 53, pp. 192-201, 2002.

[7] J. Guccione, K. Costa, and A. McCulloch, "Finite element stress analysis of left ventricular mechanics in the beating dog heart," J Biomech, vol. 28, no. 10, pp. 1167-1177, 1995. 